\title{
Binaries discovered by the SPY project
}

\section{HE 2209-1444: A massive, short period double degenerate ${ }^{\star \star \star, \star \star \star}$}

\author{
C. A. Karl ${ }^{1}$, R. Napiwotzki ${ }^{1}$, G. Nelemans ${ }^{2}$, N. Christlieb $^{3}$, D. Koester ${ }^{4}$, U. Heber $^{1}$, and D. Reimers ${ }^{3}$ \\ 1 Dr.-Remeis-Sternwarte, Astronomisches Institut der Universität Erlangen-Nürnberg, Sternwartstr. 7, 96049 Bamberg, \\ Germany \\ 2 Institute of Astronomy, Madingley Road, CB3 OHA, Cambridge, UK \\ 3 Hamburger Sternwarte, Universität Hamburg, Gojenbergsweg 112, 21029 Hamburg, Germany \\ ${ }^{4}$ Institut für Theoretische Physik und Astrophysik, Universität Kiel, 24098 Kiel, Germany
}

Received 20 March 2003 / Accepted 12 August 2003

\begin{abstract}
In the course of our search for double degenerate (DD) binaries as potential progenitors of type Ia supernovae with the UVES spectrograph at the ESO VLT (ESO SN Ia Progenitor surveY - SPY) we discovered HE 2209-1444 to be a double-lined system consisting of two DA white dwarfs. From the analysis of the radial velocity curve we determined the period of the system to be $P=6^{\mathrm{h}} 38^{\mathrm{m}} 47^{\mathrm{s}}$. The semi-amplitudes for both individual components are $109 \mathrm{~km} \mathrm{~s}^{-1}$ each. A model atmosphere analysis enabled us to derive individual temperatures for both components $(8490 \mathrm{~K}$ and $7140 \mathrm{~K}$, resp.) and masses of $0.58 M_{\odot}$ for each component. The total mass of the system is $1.15 \pm 0.07 M_{\odot}$. The system will lose angular momentum due to gravitational wave radiation and therefore will merge within 5 Gyrs - less than a Hubble time. HE 2209-1444 is the second massive, short period double degenerate detected by SPY. Its total mass is about $20 \%$ below the Chandrasekhar mass limit and therefore it does not qualify as a potentional SN Ia progenitor. However, together with our previous detections it supports the view that Chandrasekhar mass systems do exist.
\end{abstract}

Key words. stars: binaries: close - stars: supernovae: general - stars: white dwarfs

\section{Introduction}

Supernovae of type Ia (SN Ia) play an outstanding role in the study of cosmic evolution. In particular, they are regarded as one of the best standard candles for the determination of the cosmological parameters $H_{0}, \Omega$, and $\Lambda$ (e.g. Riess et al. 1998; Leibundgut 2001). However, the nature of their progenitors remains a mystery (e.g. Livio 2000).

There is general consensus that the $\mathrm{SN}$ event is due to the thermonuclear explosion of a white dwarf likely when the Chandrasekhar limit of $\approx 1.4 M_{\odot}$ is reached. But the

Send offprint requests to: C. A. Karl,

e-mail: karl@sternwarte. uni-erlangen.de

* Based on observations collected at the German-Spanish Astronomical Center (DSAZ), Calar Alto, operated by the MaxPlanck-Institute für Astronomie jointly with the Spanish National Commission for Astronomie.

$\star \star$ Based on observations at the Paranal Observatory of the European Southern Observatory for program No. 165.H-0588(A) and 167.D0407(A).

$\star \star \star$ Based on observations made with the INT operated on the island of La Palma by the Isaac Newton Group in the Spanish Observatorio del Roque de los Muchachos of the Instituto de Astrofísica de Canarias. exact nature of the progenitor system is still unclear. While it must be a binary with matter being transferred to the white dwarf from a companion, two main scenarios exists. According to the so-called double degenerate (DD) scenario (Iben \& Tutukov 1984), the mass donating companion is also a white dwarf. In the so-called singe degenerate (SD) scenario (Whelan \& Iben 1973), the mass donating component is a red giant/subgiant.

A DD has to fulfill two criteria to be considered as a potential SN Ia progenitor. The total mass of the system has to exceed the Chandrasekhar limit, and the two components have to merge in less than a Hubble time due to the loss of angular momentum via gravitational wave radiation. During the last decades, several systematic radial velocity $(R V)$ searches have been undertaken (see Marsh 2000 for a review). By now, combining all surveys nearly 180 white dwarfs have been checked for $R V$ variations yielding a sample of 18 DDs with periods of less than 6.3 days (Marsh 2000; Maxted et al. 2000a). Among these 18 systems, only 6 double-lined systems have been found (in three of them, the companion is barely detectable). However, none of the known 18 systems seems to be massive enough to qualify as a SN Ia precursor. Since theoretical simulations suggest only a few percent of all close DDs 
to be potential SN Ia progenitors (Iben et al. 1997; Nelemans et al. 2001) this result is not surprising and a larger sample size is needed to find a potential SN Ia progenitor.

Therefore - to perform a definite test of the DD scenario - we have embarked on a large spectroscopic survey of $\approx 1000$ white dwarfs using the UV-Visual Echelle Spectrograph (UVES) at the ESO VLT UT2 (Kueyen), searching for $R V$ variations of white dwarfs and pre white dwarfs (ESO SN Ia Progenitor surveY - SPY). The ongoing SPY project already provided a wealth of new $R V$ variable DDs (Napiwotzki et al. 2001a, 2003). In particular, more than a dozen doublelined systems have been found by SPY. These are of outstanding importance because the radial velocity curves can be solved for all system parameters if the gravitational redshifts of the components are unequal (see Sect. 3). An analysis of the subdwarf B plus white dwarf system HE 1047-0436 was presented in the first paper of this series (Napiwotzki et al. 2001b). In Paper II (Napiwotzki et al. 2002) we have presented the double-lined system HE 1414-0848, which has a system mass of $1.26 M_{\odot}$, but will merge only in two Hubble times.

In this paper we report on the results of our follow-up spectroscopy of the new double-lined system HE 2209-1444. We will show that this system is another massive DD, but with an orbital period shorter than the HE 1414-0848 system. After a description of the observations and data reduction in Sect. 2 we deal with the determination of the system's radial velocity curves and orbital parameters in Sect. 3. Section 4 gives a model atmosphere analysis of the spectra. We compare the results to predictions of population synthesis models in Sect. 5 and summarize in a final Sect. 6.

\section{Observations and data analysis}

HE 2209-1444 $\quad\left(\alpha_{2000}=22^{\mathrm{h}} 12^{\mathrm{m}} 18.1^{\mathrm{s}}, \quad \delta_{2000}=-14^{\circ} 29^{\prime} 48^{\prime \prime}\right.$, $B_{\mathrm{pg}}=15.3 \mathrm{mag}$ ) was discovered by the Hamburg ESO survey (HES; Wisotzki et al. 2000; Christlieb et al. 2001) as a potential cool white dwarf and, therefore, was included in our SPY project. From the first survey spectrum taken in December 2000 we found HE2209-1444 to be a double-lined binary system consisting of two DA white dwarfs. The $\mathrm{H}_{\alpha}$ line cores of both components were separated by $4.5 \AA$ in the discovery spectrum, corresponding to a $R V$ difference of $200 \mathrm{~km} \mathrm{~s}^{-1}$.

Therefore we observed the system during our follow-up observations for the SPY project at the Calar Alto Observatory, Spain. From 2001 July 6th to July 15 th a total of 15 medium resolution spectra were obtained with the TWIN spectrograph at the $3.5 \mathrm{~m}$ telescope. For the blue arm we used grating No. 5 while No. 6 was used in the red. Both gratings have 1200 lines/mm, giving a dispersion of $0.54 \AA$ /pix. The wavelength coverage ranges from $3900 \AA$ to $5000 \AA$ at a resolution of $1.3 \AA$ in the blue part of the spectrum. In the red part, the wavelength scale ranges from $6000 \AA$ to $7000 \AA$ at a resolution of $1.2 \AA$. The data were reduced with a set of standard routines for long slit spectra from the ESO MIDAS package. A two dimensional bias correction was done for the object data as well as for the dome flats. After flatfield correction, the spectra were extracted from the two dimensional images with respect to the

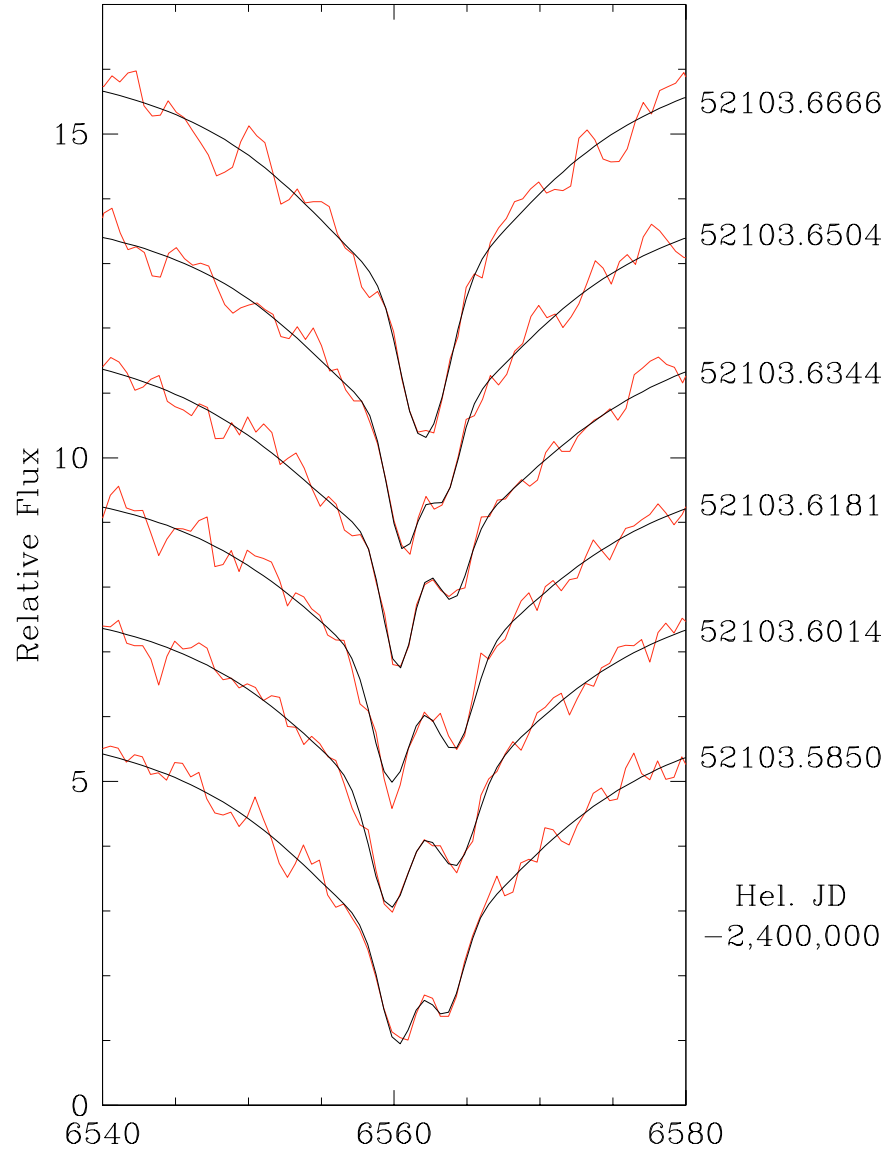

Fig. 1. Spectra of HE 2209-1444 taken at the $3.5 \mathrm{~m}$ telescope of the Calar Alto Observatory during one night. The observed spectra are as well plotted as the fits. The JD is heliocentric corrected and computed for the mid of the exposure.

sky background. Finally we performed the wavelength calibration using the internal ThAr lamp of the spectrograph.

Figure 1 shows a sequence of six $\mathrm{H}_{\alpha}$ spectra taken at Calar Alto Observatory during two hours. The observed spectra are plotted as well as the fits (cf. Sect. 3). The rapid change of the spectral appearance due to the orbital motion is obvious.

Our TWIN spectra are supplemented by spectra taken with the ESO VLT and the Isaac Newton Telescope. The UVES data we got from the ESO VLT UT2 were taken in December 2000 and October 2001. Although the data provided by the UVES reduction pipeline were quite useful for candidate selection, in many cases the quality of the pipeline data was insufficient for a quantitative analysis of Balmer line profiles. Therefore, the raw data were reduced with a semi-automatic reduction package developed in Bamberg (details will be published in a forthcoming paper).

The last set of data used for our analysis of HE 2209-1444 was taken on October 28th to October 30th using the $2.5 \mathrm{~m}$ Isaac Newton Telescope (INT) on La Palma. Eight spectra were taken with the Intermediate Dispersion Spectrograph (IDS), using the $500 \mathrm{~mm}$ camera and R1200R grating, giving a dispersion of $0.37 \AA /$ pix from 6300 to $6700 \AA$ at a resolution of $1 \AA$. The spectra were reduced (using the overscan bias level and a 
tungsten flat field) with standard IRAF $^{1}$ tasks, using optimal extraction (Horne 1986). The wavelength calibration was done using $\mathrm{CuAr}$ and CuNe lamp taken at the same position of the telescope and extracted at the same place on the CCD as the object spectra, typically giving an rms scatter less than $0.01 \AA$.

\section{Radial velocity curve and orbital parameters}

$R V \mathrm{~s}$ were determined from the TWIN and UVES spectra from a fit of a set of mathematical functions to the observed line profiles within the MIDAS context. We concentrated on a $100 \AA$ wide range of the spectra centered on $\mathrm{H}_{\alpha}$. Two Gaussians were used to fit both line cores, a Lorentzian to model the line wings and a linear function to reproduce the overall spectral trend. Radial velocities of both components were calculated from the central wavelengths of the fitted Gaussians. Afterwards the measured $R V \mathrm{~s}$ were corrected to heliocentric values. The INT spectra were analyzed using the MOLLY software package, fitting three Gaussians to the central part of the $\mathrm{H}_{\alpha}$ line.

Both components are very similar, but the left one in Fig. 1 is slightly deeper and broader. This component is called component A further on. However, sometimes it was difficult to do an unambiguous identification of the components especially near conjunction phases and in some spectra with relatively low $S / N$ ratio and of lower resolution (from Calar Alto and La Palma). Therefore, we computed the separation of the components first and fitted sine curves for a range of periods to the measured $R V$ differences and determined the $\chi^{2}$ value for each period. All times were heliocentric corrected and calculated for the center of the exposure. Doing this we produced a "difference power spectrum" indicating the quality of our $R V$ fit as a function of period (like the one presented in Fig. 2). This way we got a first estimate of the period of the system and were subsequently able to identify the individual components unambiguously for all of our spectra. This enabled us to compute for each individual component a "power spectrum". Both power spectra showed an outstanding peak at $6^{\mathrm{h}} 38^{\mathrm{m}} 46.2^{\mathrm{s}}$ $\left(0.276924^{\mathrm{d}}\right)$. An inspection of the phased $R V$ curves created using periods corresponding to other peaks in the power spectra allowed us to rule out aliases, because of one or more strongly deviating $R V$ measurements. This way, the period corresponding to the main peak of the power spectrum remained as an unambiguous solution for component $\mathrm{A}$ as well as for component B - exactly as it should be for a binary system (see Fig. 3). Finally, adding the $\chi^{2}$ values of the two individual power spectra we produced a "combined power spectrum" for the whole system (Fig. 2).

In a final step we determined accurate orbital parameters from a simultaneous fit of all spectra with the program FITSB2 (see Sect. 4 and Napiwotzki et al. 2003) in a fashion similar to the method described in Maxted et al. (2002). Two model profiles, one for each star, consisting of a combination of two

${ }^{1}$ IRAF is distributed by the National Optical Astronomy Observatories, which are operated by the Association of Universities for Research in Astronomy, Inc., under cooperative agreement with the National Science Foundation.

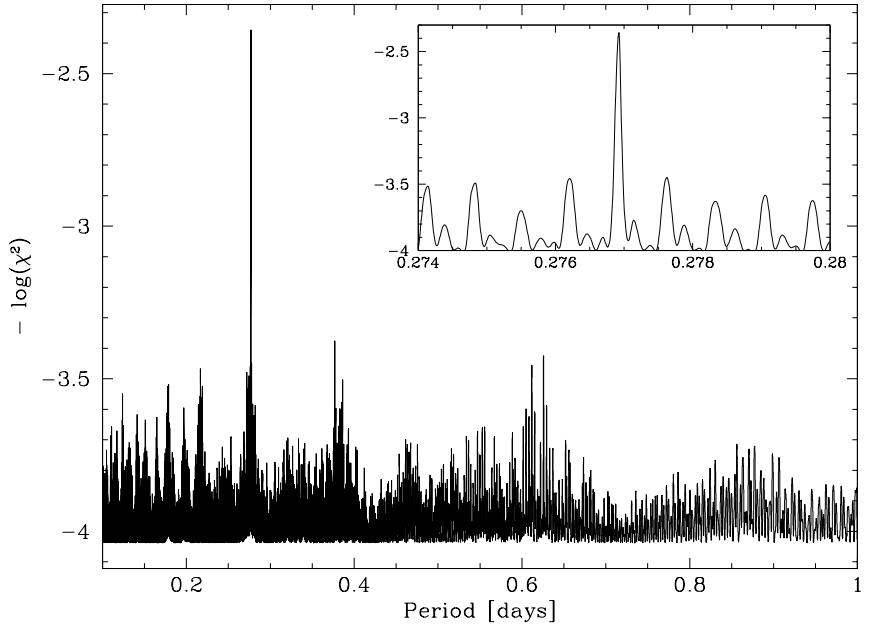

Fig. 2. Combined power spectrum of the system HE 2209-1444. The $\chi^{2}$ values obtained from the individual power spectra of the individual components are coadded.

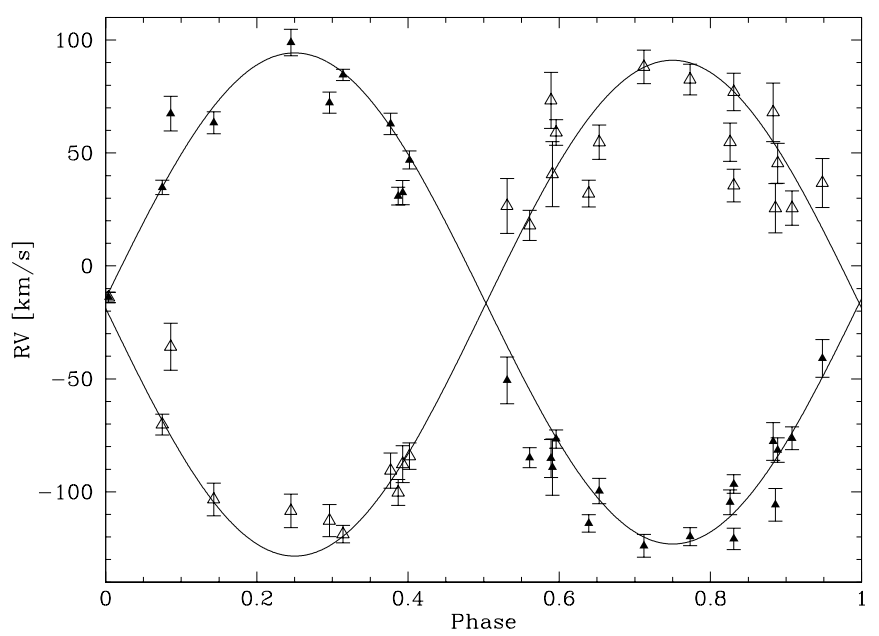

Fig. 3. Radial velocity curve of the system HE 2209-1444. The measured $R V \mathrm{~s}$ for component A (filled triangles) and $\mathrm{B}$ (open triangles) are plotted against the orbital phase.

Gaussians for the line core and a Lorentzian for the line wings were fitted. The position of each profile is determined from

$R V_{\mathrm{A} / \mathrm{B}}=\gamma_{\mathrm{A} / \mathrm{B}} \pm K_{\mathrm{A} / \mathrm{B}} \sin \left(\frac{T-T_{0}}{P}\right)$.

Free parameters are the mean velocities (including gravitational redshift) of each component $\gamma_{\mathrm{A} / \mathrm{B}}$, the (projected) orbital velocities $K_{\mathrm{A} / \mathrm{B}}$, the zeropoint $T_{0}$, and the period $P$, plus the parameters defining the line profiles.

The final ephemeris of the system for the time $T_{0}$ defined as the conjunction time at which star A moves from the blue side of the $R V$ curve to the red one (i.e. star $\mathrm{A}$ is closest to the observer) is

$$
\begin{aligned}
\operatorname{Hel} . J D\left(T_{0}\right)= & (2452097.0349 \pm 0.0021) \\
& +(0.276928 \pm 0.000006) \times E .
\end{aligned}
$$

Also from the $R V$ curves (see Fig. 3) of the FITSB2 analysis we found the semi-amplitude of component A to be $K_{\mathrm{A}}=108.7 \pm$ $7.5 \mathrm{~km} \mathrm{~s}^{-1}\left(\gamma_{\mathrm{A}}=-14.4 \pm 4.5 \mathrm{~km} \mathrm{~s}^{-1}\right)$ whereas for component $\mathrm{B}$ 
the semi-amplitude is $K_{\mathrm{B}}=109.1 \pm 10.75 \mathrm{~km} \mathrm{~s}^{-1}\left(\gamma_{\mathrm{B}}=-18.7 \pm\right.$ $\left.6.8 \mathrm{~km} \mathrm{~s}^{-1}\right)$. The mass ratio can be computed from the ratio of the semi-amplitudes to be

$\frac{M_{\mathrm{B}}}{M_{\mathrm{A}}}=\frac{K_{\mathrm{A}}}{K_{\mathrm{B}}}=1.00 \pm 0.13$

i.e. the masses of both white dwarfs are identical within the error limits. For white dwarfs the gravitational redshift can easily be measured from high resolution spectra (e.g. Reid 1996). As described in Paper II for the case of HE 1414-0848, in a double degenerate system the difference of gravitational redshifts can be measured from $\gamma_{\mathrm{A}}-\gamma_{\mathrm{B}}$. If this difference is non zero we obtain an additional constraint equation, which allows to solve for the individual masses. However, since the masses of both components of HE 2209-1444 are very similar, the redshift difference is zero within error limits. Hence we cannot solve for the individual masses from the radial velocity curve alone. The required additional information, however, will be obtained from a quantitative spectral analysis described in the next section.

\section{Spectroscopic analysis}

Since in the case of HE 2209-1444 mass estimates from the $R V$ curves alone are impossible, we had to rely on a model atmosphere analysis. Because this system is double-lined the spectra are a superposition of both individual white dwarf spectra. This problem is sometimes circumvented by assuming that the results of a simple single-lined model fit is representative for the average parameters. In Paper II we estimated the mean gravity and individual temperatures of the components of HE 1414-0848 from the analysis of individual spectra taken close to conjunction and quadrature phases. However, this approach is of limited accuracy, only.

A direct approach would be to disentangle the observed spectra by deconvolution techniques into the spectra of the individual components. Then we could analyze the spectra by fitting synthetic spectra developed for single-lined white dwarfs to the individual line profiles. Such a disentangeling procedure has been developed by Simon \& Sturm (1994) and was sucessfully applied to main sequence double-lined binaries. However, it has not been tested for white dwarfs, for which the wavelength shifts caused by orbital motions are much smaller than the line widths of the broad Balmer lines. Therefore we choose a different approach for our analysis of the HE2209-1444 system. We used the program FITSB2 (Napiwotzki et al. 2003), which performs a spectral analysis of both components of double-lined systems. It is based on a $\chi^{2}$ minimization technique using a simplex algorithm. The fit is performed on all available spectra covering different spectral phases simultaneously. For HE 1414-0848 (Paper II) we made use of spectra taken at conjunction or quadrature only. But now, all available spectral information is combined into the parameter determination procedure. An application of the program FITSB2 to HE 1414-0848 yielded encouraging results (Napiwotzki et al. 2003). A detailed description of the program and an evaluation of its performance will follow in a subsequent paper (Napiwotzki, in prep.).
A large grid of synthetic spectra for DA white dwarfs computed from LTE model atmospheres with a code described in Finley et al. (1997) was used for the analysis. A simultaneous fit of the Balmer lines $\mathrm{H}_{\beta}$ to $\mathrm{H}_{8}$ (UVES spectra) or $\mathrm{H}_{\beta}$ to $\mathrm{H}_{\delta}$ (TWIN spectra) was performed. We did not include the INT spectra for the model atmosphere analysis, because these spectra cover only the $\mathrm{H}_{\alpha}$ range. For details refer to Koester et al. (2001 and references therein). The model spectra were convolved with Gaussians with FWHMs corresponding to the resolution of the observed spectra.

The total number of fit parameters (stellar and orbital) is high. Therefore we fixed as many parameters as possible before performing the model atmosphere analysis. We have kept the radial velocities of the individual components fixed according to the radial velocity curve presented in Sect. 3. Since the mass ratio is already accurately determined from the radial velocity curve we fixed the gravity ratio. The remaining fit parameters are the effective temperatures of both components and the gravity of the primary. The gravity of the secondary is adjusted according to the primary value during the fitting procedure. The surface gravities also determine the relative weight of the two model spectra from the radius, obtained from the mass-radius relation of Benvenuto \& Althaus (1999). The flux ratio in the $V$-band is calculated from the actual parameters and the model fluxes are scaled accordingly. The individual contributions are updated consistently as part of the iteration procedure.

Strong NLTE cores are present in $\mathrm{H}_{\alpha}$ and $\mathrm{H}_{\beta}$, which cannot be reproduced by our LTE model spectra. In principle, these lines are important for the temperature determination, especially of the cooler and fainter B component. However, due to the large NLTE effects we had to exclude $\mathrm{H}_{\alpha}$ completely and the core of $\mathrm{H}_{\beta}( \pm 4 \AA$ ). The final fit results are summarized in Table 2 and a sample fit is shown in Fig. 4. The fit of the line cores is not perfect, even after excluding $\mathrm{H}_{\alpha}$. The parameter which is most sensitive to details of the fitting procedure is, as may be expected, the temperature of the fainter component $\mathrm{B}$. If, e.g., we include the wings of the $\mathrm{H}_{\alpha}$ line we increase $T_{\text {eff }}$ of component B by almost $500 \mathrm{~K}$, while the temperature of $\mathrm{A}$ and the gravity are only modified within the error limits. Thus, while the temperature of $\mathrm{B}$ is less well determined than that of A, the basic properties of the HE 2209-1444 system are reliable. Both components have identical masses $\left(M_{\mathrm{A}}=M_{\mathrm{B}}=\right.$ $\left.0.58 M_{\odot}\right)$ but different temperatures $(8490 \mathrm{~K}$ vs. $7140 \mathrm{~K})$. White dwarf masses were computed from the mass-radius relations of Benvenuto \& Althaus (1999) with "thick hydrogen envelopes" $\left(M_{\mathrm{H}} / M_{\mathrm{WD}}=10^{-4}\right)$. This result does not depend much on the choice of a particular model computation. From the thick envelope cooling sequences of Wood (1995) we derived virtually identical results: $0.58 M_{\odot}$ and $0.57 M_{\odot}$, respectively. However, since the HE 2209-1444 system is the result of a common envelope evolution it is not clear which envelope layer is the correct one. Using the "thin" envelope models $\left(M_{\mathrm{H}}=0\right)$ of Benvenuto \& Althaus (1999) as the other extreme would yield slightly lower masses $\left(0.57 M_{\odot}\right.$ and $0.56 M_{\odot}$, resp.). The resulting sum of masses of the HE 2209-1444 system is $1.15 \pm 0.07 M_{\odot}$. The error limit for the sum of masses is smaller than expected from a simple combination of the 
Table 1. Fitted heliocentric radial velocities. The heliocentric corrected JDs are given for the center of the exposures. The phases $\Phi$ are computed from the ephemeris given in Eq. (1). At $\Phi=0.982$ and $\Phi=0.040$ the individual $R V$ s of both components can not be measured accurately, because the spectra were taken close to conjunction. However, these spectra were used to determine the orbital parameters using the procedure described in Sect. 3.

\begin{tabular}{|c|c|c|c|c|c|}
\hline \multirow{2}{*}{$\begin{array}{c}\text { Hel. JD } \\
-2400000\end{array}$} & \multirow[t]{2}{*}{$\bar{\Phi}$} & \multicolumn{2}{|c|}{$\overline{\text { Heliocentric } R V[\mathrm{~km} / \mathrm{s}]}$} & \multirow[t]{2}{*}{ Telescope } & \multirow[t]{2}{*}{$\overline{\text { Instrument }}$} \\
\hline & & A & B & & \\
\hline 51885.5488 & 0.314 & $85 \pm 3$ & $-119 \pm 4$ & ESO UT2 & UVES \\
\hline 52097.5404 & 0.826 & $-105 \pm 6$ & $55 \pm 9$ & CA $3.5 \mathrm{~m}$ & TWIN \\
\hline 52097.6125 & 0.086 & $67 \pm 8$ & $-36 \pm 10$ & CA $3.5 \mathrm{~m}$ & TWIN \\
\hline 52101.5702 & 0.377 & $63 \pm 5$ & $-91 \pm 8$ & CA $3.5 \mathrm{~m}$ & TWIN \\
\hline 52101.6309 & 0.596 & $-77 \pm 4$ & $59 \pm 6$ & CA $3.5 \mathrm{~m}$ & TWIN \\
\hline 52102.5410 & 0.883 & $-78 \pm 8$ & $68 \pm 13$ & CA $3.5 \mathrm{~m}$ & TWIN \\
\hline 52102.5685 & 0.982 & - & - & CA $3.5 \mathrm{~m}$ & TWIN \\
\hline 52102.5845 & 0.040 & - & - & CA $3.5 \mathrm{~m}$ & TWIN \\
\hline 52103.5514 & 0.531 & $-51 \pm 10$ & $27 \pm 12$ & CA $3.5 \mathrm{~m}$ & TWIN \\
\hline 52103.5678 & 0.591 & $-89 \pm 12$ & $41 \pm 14$ & CA $3.5 \mathrm{~m}$ & TWIN \\
\hline 52103.5850 & 0.653 & $-100 \pm 6$ & $55 \pm 8$ & CA $3.5 \mathrm{~m}$ & TWIN \\
\hline 52103.6014 & 0.712 & $-124 \pm 5$ & $88 \pm 7$ & CA $3.5 \mathrm{~m}$ & TWIN \\
\hline 52103.6182 & 0.773 & $-120 \pm 4$ & $83 \pm 7$ & CA $3.5 \mathrm{~m}$ & TWIN \\
\hline 52103.6344 & 0.831 & $-97 \pm 4$ & $77 \pm 8$ & CA $3.5 \mathrm{~m}$ & TWIN \\
\hline 52103.6504 & 0.889 & $-82 \pm 5$ & $45 \pm 9$ & CA $3.5 \mathrm{~m}$ & TWIN \\
\hline 52103.6666 & 0.948 & $-41 \pm 8$ & $37 \pm 11$ & CA $3.5 \mathrm{~m}$ & TWIN \\
\hline 52104.6750 & 0.589 & $-85 \pm 8$ & $73 \pm 12$ & CA $3.5 \mathrm{~m}$ & TWIN \\
\hline 52105.6591 & 0.143 & $63 \pm 5$ & $-103 \pm 7$ & CA $3.5 \mathrm{~m}$ & TWIN \\
\hline 52106.5183 & 0.245 & $99 \pm 6$ & $-108 \pm 7$ & CA $3.5 \mathrm{~m}$ & TWIN \\
\hline 52172.6372 & 0.004 & $-14 \pm 2$ & $-14 \pm 2$ & ESO UT2 & UVES \\
\hline 52194.5343 & 0.075 & $35 \pm 3$ & $-70 \pm 5$ & ESO UT2 & UVES \\
\hline 52194.6249 & 0.402 & $47 \pm 4$ & $-84 \pm 6$ & ESO UT2 & UVES \\
\hline 52211.3592 & 0.831 & $-121 \pm 5$ & $36 \pm 7$ & INT & IDS \\
\hline 52211.3807 & 0.908 & $-76 \pm 5$ & $26 \pm 8$ & INT & IDS \\
\hline 52212.3189 & 0.296 & $72 \pm 5$ & $-112 \pm 7$ & INT & IDS \\
\hline 52212.3440 & 0.387 & $31 \pm 4$ & $-100 \pm 6$ & INT & IDS \\
\hline 52212.3921 & 0.561 & $-85 \pm 4$ & $18 \pm 7$ & INT & IDS \\
\hline 52212.4138 & 0.639 & $-114 \pm 4$ & $32 \pm 6$ & INT & IDS \\
\hline 52212.4822 & 0.886 & $-106 \pm 7$ & $26 \pm 11$ & INT & IDS \\
\hline 52213.4533 & 0.393 & $32 \pm 5$ & $-88 \pm 8$ & INT & IDS \\
\hline
\end{tabular}

individual errors, because the mass errors of the individual components are partially anti-correlated.

\section{Evolutionary status}

From the temperatures derived above we can estimate the ages of both components using cooling tracks for white dwarfs. Our estimate is based on the calculations of Benvenuto \& Althaus (1999) for C/O white dwarfs with thick hydrogen envelopes. Ages were interpolated from these tracks for the derived masses and temperatures of the white dwarfs. Resulting ages are 0.9 Gyrs for A and 1.4 Gyrs for B, i.e. the cooling ages of both white dwarfs differ by 0.5 Gyrs or $50 \%$.

The separation between both white dwarfs is quite small, only $1.87 R_{\odot}$. Thus HE $2209-1444$ obviously underwent phases of strong binary interaction in its history. From the size of the orbits and the period the orbital velocity can be computed to be $171 \mathrm{~km} \mathrm{~s}^{-1}$. The comparison with the observed $R V$ amplitudes allows us to determine the inclination of this system as $i=40^{\circ}$. This system will merge within 5 Gyrs due to gravitational wave radiation. Figure 5 shows the total masses and periods of the sample of known double-lined DD systems. As can be seen,
Table 2. Results of the model atmosphere analysis with FITSB2. The error limits include the uncertainties of orbital parameter determination.

\begin{tabular}{l|ccc|r}
\hline \hline comp. & $T_{\text {eff }} / \mathrm{K}$ & $\log g$ & $M / M_{\odot}$ & Age/Gyrs \\
\hline $\mathrm{A}$ & $8490 \pm 80$ & $7.97 \pm 0.05$ & $0.58 \pm 0.03$ & 0.9 \\
$\mathrm{~B}$ & $7140 \pm 110$ & $7.97 \pm 0.13$ & $0.58 \pm 0.08$ & 1.4 \\
\hline
\end{tabular}

neither HE 2209-1444 nor HE 1414-0848 (Paper II) qualify as a SN Ia progenitor. However, both objects found by SPY are much closer to the region where such progenitors are expected than any DD system known before.

We can compare the properties of HE 2209-1444 with the outcome of computations for the synthesis of the population of double white dwarfs of Nelemans et al. (2001). Figure 6 shows the expected period-mass ratio distribution of systems in which one component has a mass above $0.50 M_{\odot}$ from model A 2 of Nelemans et al. (2001). Only systems with a luminosity ratio between the components of less than 5 are plotted. The derived period and mass ratio of HE 2209-1444 is marked in Fig. 6 with its error bar. The figure shows that such systems are 


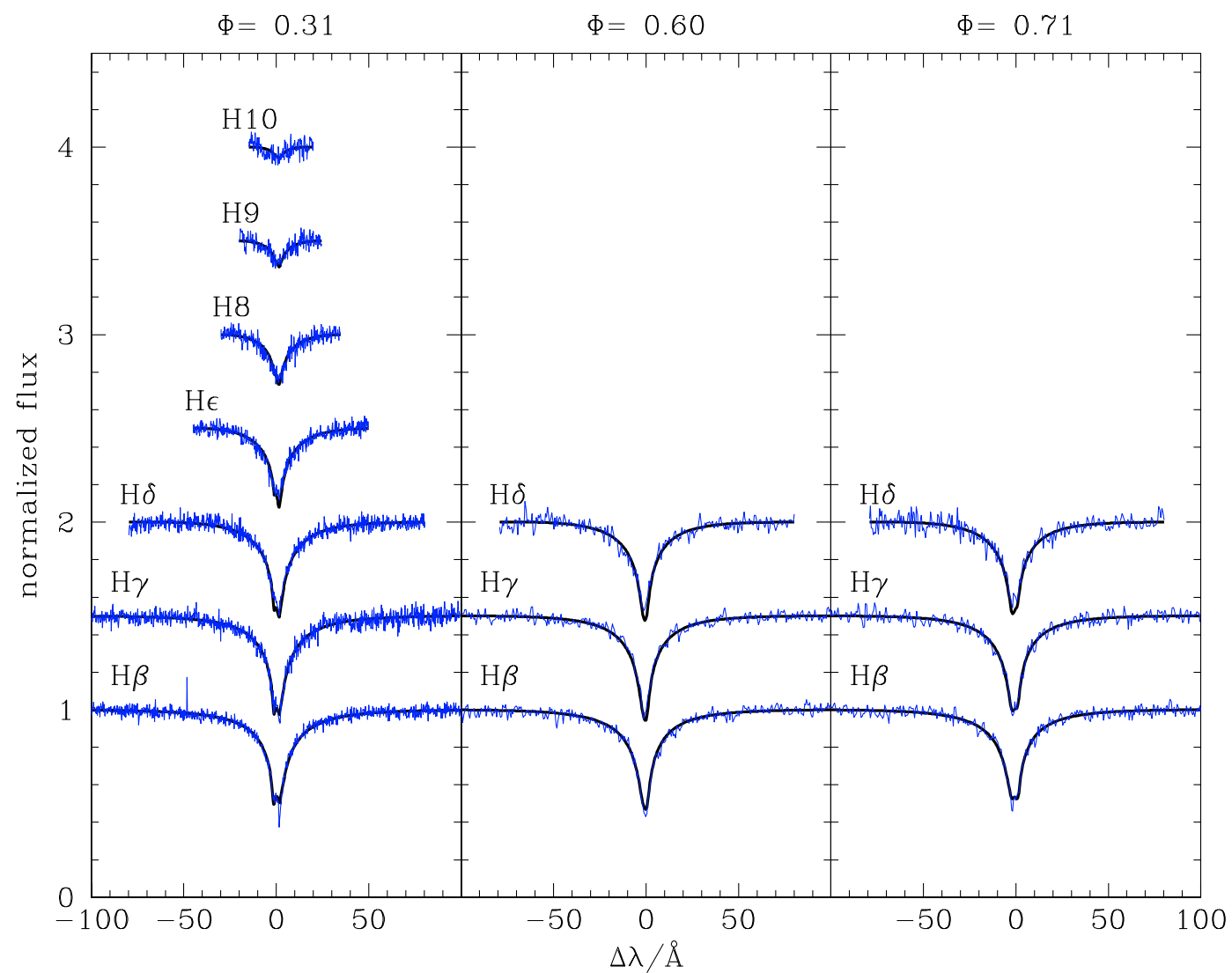

Fig. 4. Sample model atmosphere fits of HE 2209-1444 phase spectra taken at different phases: The discovery spectrum $(\Phi=0.31)$ taken with the UVES spectrograph and two TWIN spectra $(\Phi=0.60$ and $\Phi=0.71)$.

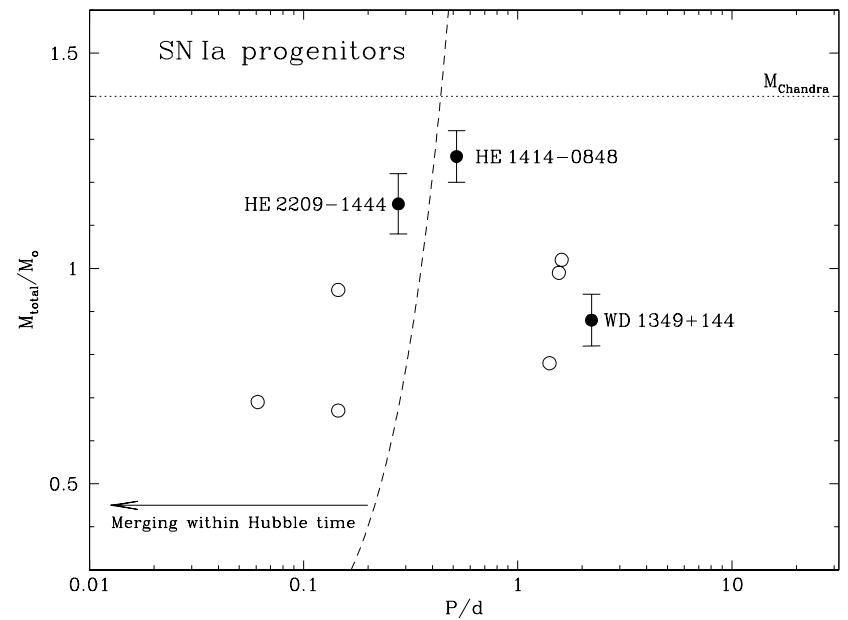

Fig. 5. Mass-period diagram of known double-lined DD systems with mass determinations for both components. Literature values from Maxted et al. $(1999,2000 \mathrm{c})$ are plotted as open circles while SPY results are shown as filled circles with error bars (WD 1349 +144 parameters from Karl et al. 2003). The Chandrasekhar limit and the region of DDs, which will merge within a Hubble time are indicated.

expected to have periods smaller than $\approx 10 \mathrm{~h}$ and either similar or more massive companions.

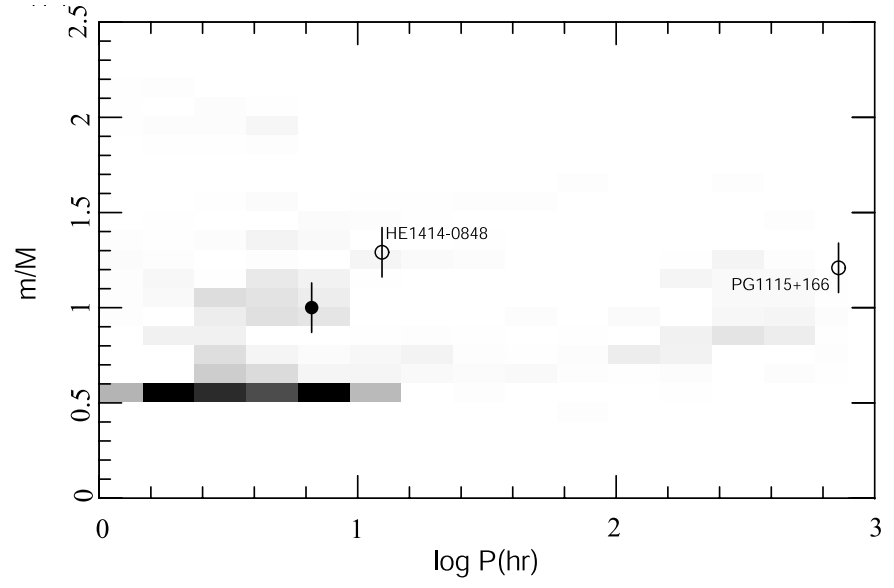

Fig. 6. Period-mass ratio distribution for the current population of DDs in the Galaxy for systems in which at least one white dwarf has a mass above $0.5 M_{\odot}$ from model A2 of Nelemans et al. (2001). Only systems with a luminosity ratio between the components of less than 5 are plotted. Grey shades indicate the expected number of systems brighter than $\mathrm{V}=15.5^{\mathrm{m}}$. The derived period and mass ratio of HE 2209-1444 is plotted as a filled dot with an error bar. Also plotted are HE 14140848 (Paper II) and PG $1115+166$ (Bergeron et al. 2002; Maxted et al. 2002).

HE 2209-1444 confirms the trend that double white dwarfs tend to have roughly equal masses, in contrast to population models using a standard common envelope description (see 
for a discussion Maxted et al. 2000c; Nelemans et al. 2001) and more in line with the results presented in Nelemans et al. (2001), in which the outcome of dynamical unstable mass transfer is calculated from the angular momentum balance, rather than the energy balance. However, it should be noted that a model using standard common envelope also produces systems similar to HE 2209-1444.

The SPY project will provide a large, homogeneous sample of close double white dwarfs, which will enable us to confirm or refuse the alternative common envelope formalism.

\section{Summary}

We report the discovery of the close double-lined binary HE 2209-1444 by SPY. From follow-up observations at three different telescopes we derived the radial velocity curve. The analysis yielded an orbital period of $P=6^{\mathrm{h}} 38^{\mathrm{m}} 47^{\mathrm{s}}$ and identical semi-amplitudes of $109 \mathrm{~km} \mathrm{~s}^{-1}$ for both components. Parameters of both individual components were determined from a model atmosphere analysis. We made use of a novel method which combines all available spectra covering the different orbital phases for a simultaneous fit. The result is a common gravity of 7.97 and temperatures of $8490 \mathrm{~K}$ and $7140 \mathrm{~K}$ for components $\mathrm{A}$ and $\mathrm{B}$, respectively.

Masses were determined from a subsequent comparison with the cooling tracks of Benvenuto \& Althaus (1999). The results are masses of $0.58 M_{\odot}$ for the individual components and a total mass for the HE 2209-1444 system of $1.15 \pm 0.07 M_{\odot}$. This makes HE 2209-1444 the second most massive DD ever found in a $R V$ survey, surpassed only by HE 1414-0848, another detection of the SPY project (Napiwotzki et al. 2002). However, the separation of the components in HE 2209-1444 is smaller than in the case of HE 1414-0848. Therefore, the merging time of the former system is 5 Gyrs whereas in the latter case the system will merge within two Hubble times.

We compared the resulting orbital parameters of HE 2209-1444 with the outcome of theoretical calculations for the formation of DDs and find that the properties of HE 2209-1444 are in agreement with recent predictions.

Acknowledgements. C. A. K. is supported by DFG under grant Na365/3-1 and by travel grants. G. N. would like to thank T. Marsh for the use of his MOLLY and aliases software.

\section{References}

Benvenuto, O. G., \& Althaus, L. G. 1999, MNRAS, 303, 30

Bergeron, P., \& Liebert, J. 2002, ApJ, 566, 1091

Christlieb, N., Wisotzki, L., Reimers, D., et al. 2001, A\&A, 356, 898

Finley, D. S., Koester, D., \& Basri, G. 1997, ApJ, 488, 375

Horne, K. 1986, PASP, 98, 609

Iben, I. Jr., \& Tutukov, A. V. 1984, ApJS, 54, 335

Iben, I. Jr., Tutukov, A. V., \& Yungelson, L. R. 1997, ApJ, 475, 291

Karl, C. A., Napiwotzki, R., Heber, U., et al. 2003, in White Dwarfs, ed. D. de Martino, R. Silvotti, J.-E. Solheim, \& R. Kalytis, NATO Science Series, 105, 43

Koester, D., Napiwotzki, R., Christlieb, N., et al. 2001, A\&A, 378, 556

Livio, M. 2000, in Type Ia Supernova: Theory and Cosmology, ed. J. C. Niemeyer, \& J. W. Truran (Cambridge Univ. Press), 33

Leibundgut, B. 2001, ARA\&A, 39, 67

Marsh, T. R. 2000, NewAR, 44, 119

Maxted, P. F. L., Burleigh, M. R., Marsh, T. R., \& Bannister, N. P. 2002, MNRAS, 334, 833

Maxted, P. F. L., \& Marsh, T. R. 1999, MNRAS, 307, 122

Maxted, P. F. L., Marsh, T. R., \& Moran, C. K. J. 2000a, MNRAS, 319,305

Maxted, P. F. L., Marsh, T. R., Moran, C. K. J., \& Han, Z. 2000c, MNRAS, 314, 334

Maxted, P. F. L., Marsh, T. R., \& Moran, C. K. J. 2002, MNRAS, 332, 745

Napiwotzki, R., Christlieb, N., Drechsel, H., et al. 2001a, AN, 322, 411

Napiwotzki, R., Christlieb, N., Drechsel, H., et al. 2003, in From Twilight to Highlight, ed. W. Hillebrandt, B. Leibundgut, ESO Astrophysics Symposia (Berlin, Heidelberg: Springer-Verlag), 134

Napiwotzki, R., Edelmann, H. Heber, U., et al. 2001b, A\&A, 378, L17 (Paper I)

Napiwotzki, R., Koester, D., Nelemans, G., et al. 2002, A\&A, 386, 957 (Paper II)

Nelemans, G., Yungelson, L. R., Portegies Zwart, S. F., \& Verbunt, F. 2001, A\&A, 365, 491

Reid, I. N. 1996, AJ, 111, 2000

Riess, A. G., Fillipenko, A. V., Challis, P., et al. 1998, AJ, 116, 1009

Simon, K. P., \& Sturm, E. 1994, A\&A, 281,286

Whelan, J., \& Iben, I. Jr. 1973, ApJ, 186, 1007

Wisotzki, L., Christlieb, N., Bade, N., et al. 2000, A\&A, 358, 77

Wood, M. A. 1995, in White Dwarfs, Proceedings of the 9th European Workshop on White Dwarfs held at Kiel, Germany, 29 August 29th to September 1st 1994, ed. D. Koester \& K. Werner (Berlin, Heidelberg, New York: Springer-Verlag), 348 